Pacific Journal of Mathematics

A SHEAF THEORETIC REPRESENTATION OF RINGS WITH 


\title{
A SHEAF THEORETIC REPRESENTATION OF RINGS WITH BOOLEAN ORTHOGONALITIES
}

\author{
Patrick N. Stewart
}

It is shown that certain associative rings with Boolean orthogonalities are isomorphic to rings of global sections.

Let $A$ be a ring and $\perp$ a relation on $A$. For each subset $S$ of $A$ define

$$
S^{\perp}=\{x \in A \mid x \perp s \quad \text { for all } s \in S\} \text { and } S^{\perp \perp}=\left(S^{\perp}\right)^{\perp} \text {. }
$$

When $S=\{s\}$ we write $s^{\perp}$ and $s^{\perp \perp}$ instead of $\{s\}^{\perp}$ and $\{s\}^{\perp \perp}$. Subsets of $A$ of the form $S^{\perp}$ are polars. The relation $\perp$ is a Boolean orthogonality if all polars are two-sided ideals and if, for all $x, y \in A$,

1. $x \perp y \rightarrow y \perp x, \quad$ 2. $x \perp x \rightarrow x=0$, and

3. $x^{\perp \perp} \cap y{ }^{\perp \perp}=(0) \rightarrow x \perp y$.

The set of polars is a Boolean algebra (see [3]) with meet and join defined by

$$
B \wedge C=B \cap C \quad \text { and } \quad B \vee C=\left(B^{\perp} \wedge C^{\perp}\right)^{\perp} .
$$

Boolean orthogonalities have been studied by Davis [3], Cornish [1] and by Cornish and Stewart [2].

Throughout this paper we shall assume that $A$ is an associative ring with an identity and with a Boolean orthogonality $\perp$. We shall also assume that the following finiteness condition is satisfied:

for any two elements $x, y \in A$ there is a finite set $F \subseteq A$ such that $x^{\perp \perp} \wedge y^{\perp \perp}=F^{\perp \perp}$.

Notice that if $F=\left\{f_{1}, \cdots, f_{n}\right\}$, then $F^{\perp}=f_{1}^{\perp} \wedge \cdots \wedge f_{n}^{\perp}$ and $F^{\perp \perp}=$ $f_{1}^{\perp \perp} \vee \cdots \vee f_{n}^{\perp \perp}$.

An ideal $I$ of $A$ is a $\perp$-ideal if $F^{\perp \perp} \subseteq I$ for every finite set $F \subseteq I$, and $I$ is $\perp$-prime if $I \neq A$ and whenever the intersection of two polars $B$ and $C$ is contained in $I$, either $B \subseteq I$ or $C \subseteq I$.

Lemma. Assume that $P$ is either a $\perp$-prime ideal or $P=A$, that $I$ is $a \perp$-ideal and that $x \in A \backslash I$ is such that $x^{\perp \perp} \wedge x^{\perp \perp} \subseteq I$ implies that $a \in P$. Then there is a $\perp$-prin $e \perp$-ideal $Q$ such that $I \subseteq Q \subseteq P$ and $x \notin Q$. 
Proof. Using Zorn's Lemma select a $\perp$-ideal $Q \supseteq I$ maximal with respect to the property " $x \notin Q$ and $x^{\perp \perp} \wedge x^{\perp \perp} \subseteq Q$ implies that $a \in P$ ". Clearly $I \subseteq Q \subseteq P$.

Suppose that $B$ and $C$ are polars neither of which is contained in $Q$. Choose $b \in B \backslash Q$ and $c \in C \backslash Q$. Then

$$
B^{\prime}=\cup\left\{F^{\perp \perp} \mid F \text { is a finite subset of }\{b\} \cup Q\right\}
$$

and

$$
C^{\prime}=\cup\left\{G^{\perp 1} \mid G \text { is a finite subset of }\{c\} \cup Q\right\}
$$

are $\perp$-ideals which properly contain $Q$. By the maximality of $Q$ either $x^{\perp \perp} \subseteq B^{\prime}$ or $x^{\perp \perp} \wedge b_{1}^{\perp \perp} \subseteq B^{\prime}$ for some $b_{1} \in A \backslash P$, and $x^{\perp \perp} \subseteq C^{\prime}$ or $x^{\perp \perp} \wedge c_{1}^{\perp \perp} \subseteq C^{\prime}$ for some $c_{1} \in A \backslash P$. Thus we obtain finite sets $\left\{b, f_{1}, \cdots, f_{n}\right\} \subseteq\{b\} \cup Q$ and $\left\{c, g_{1}, \cdots, g_{m}\right\} \subseteq\{c\} \cup Q$ such that one of $x^{\perp \perp}, x^{\perp \perp} \wedge b_{1}^{\perp \perp}, x^{\perp \perp} \wedge c_{1}^{\perp \perp}$ or $x^{\perp \perp} \wedge b_{1}^{\perp \perp} \wedge c_{1}^{\perp \perp}$ is contained in

$$
\begin{aligned}
\left\{b, f_{1}, \cdots, f_{n}\right\}^{\perp \perp} \wedge\left\{c, g_{1}, \cdots, g_{m}\right\}^{\perp \perp} & \\
& =\left(b^{\perp \perp} \vee f_{1}^{\perp \perp} \vee \cdots \vee f_{n}^{\perp \perp}\right) \wedge\left(c^{\perp \perp} \vee g_{1}^{\perp \perp} \vee \cdots \vee g_{m}^{\perp \perp}\right) \\
& =\left(b^{\perp \perp} \wedge c^{\perp \perp}\right) \vee H^{\perp \perp}
\end{aligned}
$$

where $H$ is a finite subset of $Q$ (we have used the distributivity of the Boolean algebra of polars and also the finiteness condition). If $b^{\perp \perp} \wedge c^{\perp \perp} \subseteq Q$, then $x^{\perp \perp} \subseteq Q$ or $x^{\perp \perp} \wedge l^{\perp \perp} \subseteq Q$ for some $d \in A \backslash P$ both of which contradict the choice of $Q$. Thus $B \cap C \not \subset Q$ and we conclude that $Q$ is $\perp$-prime.

For the remainder of this paper $\bar{X}$ will be fixed set of $\perp$-prime ideals which contains all $\perp$-prime $\perp$-ideals and which is full (that is, if $I$ is a sum of polars and $I \neq A$, then $I \subseteq P$ for some $P \in \underline{X}$ ).

Proposition 2. (Cornish [1]). For each $P \in \bar{X}$,

$$
\left\{x \in A \mid x^{\perp} \not \subset P\right\}=\cap\{R \in \underline{X} \mid R \subseteq P\}=\cap\{Q \in \underline{X} \mid Q \subseteq P
$$

and $Q$ is a $\perp$-prime $\perp$-ideal $\}$.

Proof. Suppose that $x^{\perp} \not \subset P$ and $R$ is a $\perp$-prime ideal contained in $P$. Then $x^{\perp \perp} \wedge x^{\perp}=(0) \subseteq R$ and so $x \in R$.

If $x^{\perp} \subseteq P$, then by Lemma 1 (take $I=x^{\perp}$ ) there is a $\perp$-prime $\perp$-ideal $Q \subseteq P$ such that $x \notin Q$. This establishes the result. 
The set described in the proposition will be denoted by $O_{p}$. We note that $O_{P}=P$ if and only if $P$ is minimal in $\bar{X}$.

Let $P \in \bar{X}$. The set $O_{P}$, being an intersection of $\perp$-ideals, is itself a $\perp$-ideal. Define a relation (also denoted by $\perp$ ) on $A / O_{P}$ by

$$
\left(x+O_{P}\right) \perp\left(y+O_{P}\right) \leftrightarrow x^{\perp \perp} \wedge y^{\perp \perp} \subseteq O_{P}
$$

This relation is well-defined because if $x_{1}=x+a$ and $y_{1}=y+b$ where $a, b \in O_{P}$, then

$$
x_{1}^{\perp \perp} \wedge y_{1}^{\perp \perp}=(x+a)^{\perp \perp} \wedge(y+b)^{\perp \perp} \subseteq\left(x^{\perp \perp} \vee x^{\perp \perp}\right) \wedge\left(y^{\perp \perp} \vee b^{\perp \perp}\right)
$$

and so $x_{1}^{\perp \perp} \wedge y_{1}^{\perp \perp} \subseteq\left(x^{\perp \perp} \wedge y^{\perp \perp}\right) \vee F^{\perp \perp}$ where $F$ is a finite subset of $O_{P}$. It is routine to check that

$$
x^{\perp}+O_{P} \subseteq\left(x+O_{P}\right)^{\perp} \text { and } x^{\perp \perp}+O_{P} \subseteq\left(x+O_{P}\right)^{\perp \perp}
$$

for each $x \in A$, and that the relation $\perp$ is a Boolean orthogonality on $A / O_{P}$.

Proposition 3. For each $P \in \bar{X}, \bar{P}=P / O_{P}$ is a $\perp$-prime ideal of $A / O_{P}$ which contains all proper polars of $A / O_{P}$.

Proof. Let $\bar{B}$ and $\bar{C}$ be polars in $A / O_{P}$ such that $\bar{B} \cap \bar{C} \subseteq \bar{P}$. Suppose that $\bar{B} \not \subset \bar{P}$. Then there is an element $b \in A$ such that $b+O_{P} \in \bar{B} \backslash \bar{P}$. Let $c+O_{P} \in \bar{C}$. Then

$$
\left(b^{\perp \perp}+O_{P}\right) \cap\left(c^{\perp \perp}+O_{P}\right) \subseteq\left(b+O_{P}\right)^{\perp \perp} \cap\left(c+O_{P}\right)^{\perp \perp} \subseteq \bar{B} \cap \bar{C} \subseteq \bar{P}
$$

and so $b^{\perp \perp} \cap c^{\perp \perp} \subseteq P$. Since $b \notin P$ we conclude that $c \in P$ and so $\bar{C} \subseteq \bar{P}$. Thus $\bar{P}$ is $\perp$-prime.

Suppose that $a^{\perp \perp} \wedge b^{\perp \perp} \subseteq O_{P}$. Then there is a finite set $\left\{f_{1}, \cdots, f_{n}\right\} \subseteq$ $O_{P}$ such that

$$
a^{\perp \perp} \wedge b^{\perp \perp}=\left\{f_{1}, \cdots, f_{n}\right\}^{\perp \perp}=f_{1}^{\perp \perp} \vee \cdots \vee f_{n}^{\perp \perp} .
$$

For each $i=1, \cdots, n, f_{i} \in O_{P}$ and so $f_{i}^{\perp} \not \subset P$. Thus $f_{1}^{\perp} \wedge \cdots \wedge f_{n}^{\perp} \not \subset P$. Also, $b^{\perp \perp} \wedge f_{1}^{\perp} \wedge \cdots \wedge f_{n}^{\perp} \subseteq a^{\perp}$ because $a^{\perp \perp} \wedge,{ }^{\perp \perp} \wedge f_{1}^{\perp} \wedge \cdots \wedge f_{n}^{\perp}=(0)$. If $a \notin O_{p}$, then $a^{\perp} \subseteq P$ and so, since $f_{1}^{\perp} \wedge \cdots \wedge f_{n}^{\perp} \not \subset P, b^{\perp 1} \subseteq P$. Thus $\bar{P}$ contains $\left(a+O_{P}\right)^{\perp}$ for all $a \notin O_{P}$. It follows that $\bar{P}$ contains all proper polars of $A / O_{P}$. 
Let $S$ be the disjoint union of the factor rings $A / O_{P}$. The relation (also denoted by $\perp$ ) on the product

defined by

$$
\begin{gathered}
\Pi\left\{A / O_{P} \mid P \in \underline{\bar{X}}\right\} \\
=\left\{f: \underline{X} \rightarrow S \mid f(P) \in A / O_{P} \text { for all } P \in \bar{X}\right\}
\end{gathered}
$$

$$
f \perp g \leftrightarrow f(P) \perp g(P) \text { in } A / O_{P} \text { for all } P \in \underline{X}
$$

is a Boolean orthogonality. Each $a \in A$ determines a function $\hat{a} \in$ $\Pi\left\{A / O_{P} \mid P \in \bar{X}\right\}$ defined by $\hat{a}(P)=a+O_{P}$. It follows from Lemma 1 that $\cap\{P \mid P$ is a $\perp$-prime $\perp$-ideal $\}=(0)$ and so $\cap\left\{O_{P} \mid P \in \underline{X}\right\}=(0)$. Thus we obtain the usual embedding

$$
A \hat{\rightarrow} \hat{A} \subseteq \Pi\left\{A / O_{P} \mid P \in \underline{X}\right\}
$$

This embedding respects orthogonalities; that is, $a \perp b$ in $A$ if and only if $\hat{a} \perp \hat{b}$ in the product.

We define a topology on $\underline{X}$ by declaring the basic open sets to be the subsets of the form

$$
\underline{X}(a)=\left\{P \in \underline{X} \mid a^{\perp \perp} \not \subset P\right\} .
$$

Notice that $\underset{X}{X}(a) \cap \underline{X}(b) \supseteq \underline{X}(c)$ for all $c \in a^{\perp \perp} \wedge b^{\perp \perp}$ and so these sets do qualify as a topological base.

Suppose that $\{\underline{X}(a) \mid a \in C\}$ is a cover of $\underline{\bar{X}}$ consisting of basic open sets. Then $\Sigma\left\{a^{\perp 1} \mid a \in C\right\}=A$ because $\bar{X}$ is full. Since $A$ has an identity there is a finite set $F \subseteq C$ such that $\Sigma\left\{a^{\perp \perp} \mid a \in F\right\}=A$. Thus $\{\underline{X}(a) \mid a \in F\}$ covers $\underline{\bar{X}}$ and so $\underline{X}$ is quasi-compact.

Give $S$ the topology generated by sets of the form $\hat{a}[U]=$ $\left\{a+O_{P} \mid P \in U\right\}$ where $U$ is open in $\bar{X}$ and $a \in A$. We obtain a sheaf of rings $(S, \pi, \bar{X})$ where $\pi: S \rightarrow \bar{X}$ is the projection onto $\bar{X}$.

Let $\Gamma=\left\{f \mid f \in \Pi\left\{A / O_{P} \mid P \in \bar{X}\right\}\right.$ is continuous $\}$ be the ring of global sections. The following observation shows that $\hat{A} \subseteq \Gamma$ : for all $x, y \in A$, $\left\{P \in \underline{X} \mid x-y \in O_{P}\right\}$ is open in $\underline{X}$. To see this notice that if $x-y \in O_{Q}$, then $Q \in \bar{X}(u) \subseteq\left\{P \in \underline{X} \mid x-y \in O_{P}\right\}$ where $u$ is any element in $(x-$ $y)^{\perp} \backslash Q$.

TheOrem 4. $\hat{A}=\Gamma$.

Proof. Let $f \in \Gamma$. Since $\bar{X}$ is quasi-compact there are finite sets $\left\{a_{1}, \cdots, a_{n}\right\}$ and $\left\{v_{1}, \cdots, v_{n}\right\}$ such that $\underline{\bar{X}}=\underline{X}\left(a_{1}\right) \cup \cdots \cup \underline{X}\left(a_{n}\right)$ and $f(P)=v_{i}+O_{P}$ for all $P \in \underline{X}\left(a_{i}\right)$.

Notice that $v_{i}-v_{j} \in \cap\left\{O_{P} \mid P \in \underline{X}\left(a_{i}\right) \cap \underline{X}\left(a_{j}\right)\right\}$, so $\left(v_{i}-v_{j}\right)^{\perp} \subseteq$ 
$Q \in \bar{X}$ implies that $a_{i}^{\perp \perp} \wedge x_{j}^{\perp \perp} \subseteq Q$. It follows from Lemma 1 (take $P=A$ and $I=\left(v_{i}-v_{j}\right)^{\perp}$ for each $\left.x \notin\left(v_{i}-v_{j}\right)^{\perp}\right)$ that

$$
\left(v_{i}-v_{j}\right)^{\perp}=\cap\left\{Q \mid\left(v_{i}-v_{j}\right)^{\perp} \subseteq Q \in \underline{X}\right\}
$$

and so $a_{i}^{\perp \perp} \wedge x_{i}^{\perp \perp} \subseteq\left(v_{i}-v_{j}\right)^{\perp}$. Thus $\left(v_{i}-v_{j}\right)^{\perp \perp} \wedge x{ }_{i}^{\perp \perp} \subseteq a_{j}^{\perp}$.

Since $\underline{\bar{X}}=\bar{X}\left(a_{1}\right) \cup \cdots \cup \underline{X}\left(a_{n}\right)$ and $\underline{X}$ is full, $a_{1}^{\perp \perp}+\cdots+a_{n}^{1 \perp}=A$. Choose $u_{i} \in a_{i}^{\perp \perp}$ such that $1=u_{1}+\cdots+u_{n}$ and let $v=u_{1} v_{1}+\cdots+u_{n} v_{n}$. Then

$$
v-v_{j}=u_{1}\left(v_{1}-v_{j}\right)+\cdots+u_{n}\left(v_{n}-v_{j}\right) \in a_{j}^{\perp} \subseteq O_{P}
$$

for all $P \in \bar{X}\left(a_{j}\right)$. Thus $f(P)=v_{j}+O_{P}=v+O_{P}$ for all $P \in \underline{X}\left(a_{j}\right)$ and so $f=\hat{v} \in \hat{A}$.

f-rings (Keimal [4]). Let $A$ be an $f$-ring with identity. The relation defined by $x \perp y \leftrightarrow|x| \wedge|y|=0$ is a Boolean orthogonality and $x^{\perp \perp} \wedge y^{\perp \perp}=(|x| \wedge y \mid)^{\perp \perp}$. Let $\bar{X}$ be the set of irreducible $\ell$-ideals. Then $\bar{X}$ is full because polars are $\ell$-ideals and sums of $\ell$-ideals are again $\ell$-ideals. Also, all $\perp$-prime $\perp$-ideals are irreducible $\ell$-ideals and so $A$ is isomorphic to the $f$-ring of all global sections of the sheaf $(S, \pi, \underline{X})$.

Reduced rings (Koh [5]). Let $A$ be a ring with identity and no nonzero nilpotent elements. The relation defined by $x \perp y \leftrightarrow x y=0$ is a Boolean orthogonality and $x^{\perp \perp} \wedge y^{\perp \perp}=(x y)^{\perp \perp}$. Let $\underline{X}$ be the set of all prime ideals of $A$. Clearly $\bar{X}$ is full. Also, all $\perp$-prime $\perp$-ideals are completely prime and so $A$ is isomorphic to the ring of global sections of the sheaf $(S, \pi, \bar{X})$. Each stalk $A / O_{P}$ is reduced (Proposition 2) and the prime ideal $P / O_{P}$ contains all zero divisors (Proposition 3).

Semiprime rings. Let $A$ be a semiprime ring with identity. The relation defined by $x \perp y \leftrightarrow(x)(y)=(0)$ is a Boolean orthogonality. However, the finiteness condition may not be satisfied as the following example shows.

Let $R$ be a semiprime ring with identity, $R^{\prime}$ the ring of $3 \times 3$ matrices with entries from $R$,

$$
x=\left[\begin{array}{lll}
0 & 1 & 0 \\
0 & 0 & 0 \\
0 & 0 & 0
\end{array}\right] \text { and } y=\left[\begin{array}{lll}
0 & 0 & 1 \\
0 & 0 & 0 \\
0 & 0 & 0
\end{array}\right] \text {. }
$$

Define $\bar{x}$ and $\bar{y}$ in $P=\Pi\left\{R_{n} \mid R_{n}=R^{\prime}\right.$ for $\left.n=1,2, \cdots\right\}$ by

$$
\begin{aligned}
& \bar{x}(n)=\left\{\begin{array}{llll}
x & \text { if } & n \equiv 1 & (\bmod 2) \\
0 & \text { if } & n \neq 1 & (\bmod 2)
\end{array}\right. \\
& \bar{y}(n)=\left\{\begin{array}{llll}
y & \text { if } & n \equiv 1 & (\bmod 3) \\
0 & \text { if } & n \neq 1 & (\bmod 3) .
\end{array}\right.
\end{aligned}
$$


Notice that $\bar{x} \bar{y}=\bar{y} \bar{x}=\bar{x}^{2}=\bar{y}^{2}=0$. Let $E$ be the subring of $P$ which is generated by the identity of $P, \bar{x}, \bar{y}$ and

$$
\begin{array}{ccc}
\Sigma\left\{R_{n} \mid R_{n}=R^{\prime} \quad\right. \text { for } & n=1,2, \cdots\} . & \text { Then } \\
\bar{x}^{\perp \perp}=\{f \in E \mid f(n)=0 & \text { for } n \neq 1 & (\bmod 2)\}, \\
\bar{y}^{\perp \perp}=\{f \in E \mid f(n)=0 & \text { for } n \neq 1 & (\bmod 3)\},
\end{array}
$$

and so

$$
\bar{x}^{\perp \perp} \wedge \bar{y}^{\perp \perp}=\{f \in E \mid f(n)=0 \text { for } n \neq \equiv 1 \quad(\bmod 6)\}
$$

If $\bar{x}^{\perp \perp} \wedge \bar{y}^{\perp \perp}=\left\{f_{1}, \cdots, f_{n}\right\}^{\perp \perp}$, then at least one of the $f_{i}$ must satisfy $f_{i}(n) \neq 0$ for infinitely many positive integers $n$. But then there are integers $\alpha, \beta$ and $\gamma$ such that $f_{i}(n)=(\alpha+\beta \bar{x}+\gamma \bar{y})(n)$ for all but a finite number of positive integers $n$. This is incompatible with the requirement that $f_{i}(n)=0$ for $n \neq 1(\bmod 6)$.

When the finiteness condition is satisfied (for instance, when $A$ satisfies the maximum condition on annihilators), $A$ is isomorphic to the ring of all global sections of the sheaf $(S, \pi, \bar{X})$ where $\underline{X}$ is the set of prime ideals of $A$. Each stalk $A / O_{P}$ is semiprime (Proposition 1) and the prime ideal $P / O_{P}$ contains all two-sided annihilator ideals of $A / O_{P}$ (Proposition 2).

\section{REFERENCES}

1. W. H. Cornish, Boolean orthogonalities and minimal primes, preprint.

2. W. H. Cornish and P. N. Stewart, Direct and subdirect decompositions of universal algebras with a Boolean orthogonality, preprint.

3. G. Davis, Rings with orthogonality relations, Bull. Austral. Math. Soc., 4 (1971), 163-178.

4. K. Keimal, The representation of lattice ordered groups and rings by sections in sheaves, Lecture Notes in Math. 248, 1-98, Springer, Berlin-Heidelberg-New York, 1971.

5. K. Koh, On functional representations of a ring without nilpotent elements, Canad. Math. Bull., 14 (1971), 349-352.

Received April 30, 1974. Part of the research for this paper was done at the 1973 Canadian Mathematical Congress Summer Research Institute in Sherbrooke, Quebec, Canada. 


\section{CONTENTS}

Zvi Artstein and John A. Burns, Integration of compact set-valued functions

J. A. Beachy and W. D. Blair, Rings whose faithful left ideals are cofaithful

Mark Benard, Characters and Schur indices of the unitary reflection group $[321]^{3}$

H. L. Bentley and B. J. Taylor, Wallman rings ............................. 15

E. Berman, Matrix rings over polynomial identity rings II ............... 37

Simeon M. Berman, A new characterization of characteristic functions of absolutely continuous distributions ........................ 323

Monte B. Boisen, Jr. and Philip B. Sheldon, Pre-Prüfer rings ............ 331

A. K. Boyle and K. R. Goodearl, Rings over which certain modules are injective

J. L. Brenner, R. M. Crabwell and J. Riddell, Covering theorems for finite nonabelian simple groups. $V$

H. H. Brungs, Three questions on duo rings .................................... 345

Iracema M. Bund, Birnbaum-Orlicz spaces of functions on groups ....351

John D. Elwin and Donald R. Short, Branched immersions between 2-manifolds of higher topological type

J. K. Finch, The single valued extension property on a Banach space ................................................................................. 61

J. R. Fisher, A Goldie theorem for differentiably prime rings ............ 71

Eric M. Friedlander, Extension functions for rank 2, torsion free abelian groups ...................................................................... 371

J. Froemke and R. Quackenbusch, The spectrum of an equational class of groupoids

B. J. Gardner, Radicals of supplementary semilattice sums of

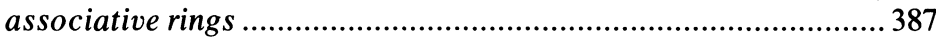

Shmuel Glasner, Relatively invariant measures ...............................393

G. R. Gordh, Jr. and Sibe Mardešić, Characterizing local connectedness in inverse limits...

S. Graf, On the existence of strong liftings in second countable topological spaces

S. Gudder and D. Strawther, Orthogonally additive and orthogonally increasing functions on vector spaces ........................................427

F. Hansen, On one-sided prime ideals .......................................... 79

D. J. Hartfiel and C. J. Maxson, A characterization of the maximal monoids and maximal groups in $\beta x$.

Robert E. Hartwig and S. Brent Morris, The universal flip matrix and the generalized faro-shuffle 


\section{Pacific Journal of Mathematics}

Vol. 58, No. 1

March, 1975

John Allen Beachy and William David Blair, Rings whose faithful left ideals are cofaithful .................................... 1

Herschel Lamar Bentley and Barbara June Taylor, Wallman rings ........ 15

Elizabeth Berman, Matrix rings over polynomial identity rings. II ...... 37

Ann K. Boyle and Kenneth R. Goodearl, Rings over which certain modules are injective ................................. 43

J. L. Brenner, Robert Myrl Cranwell and James Riddell, Covering theorems for finite nonabelian simple groups. $V \ldots \ldots \ldots \ldots \ldots \ldots \ldots \ldots \ldots$

James Kenneth Finch, The single valued extension property on a Banach

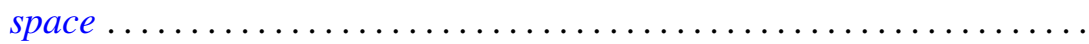

John Robert Fisher, A Goldie theorem for differentiably prime rings........ Friedhelm Hansen, On one-sided prime ideals .................... Jon Craig Helton, Product integrals and the solution of integral equations..........................................

Barry E. Johnson and James Patrick Williams, The range of a normal

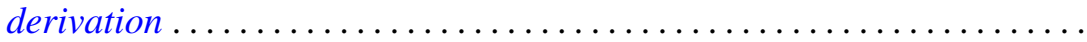

Kurt Kreith, A dynamical criterion for conjugate points ............ 123

Robert Allen McCoy, Baire spaces and hyperspaces .............. 133

John McDonald, Isometries of the disk algebra ................ 143

H. Minc, Doubly stochastic matrices with minimal permanents ......... 155

Shahbaz Noorvash, Covering the vertices of a graph by vertex-disjoint paths. ...

Theodore Windle Palmer, Jordan *-homomorphisms between reduced Banach*-algebras

Donald Steven Passman, On the semisimplicity of group rings of some

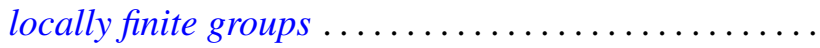

Mario Petrich, Varieties of orthodox bands of groups .

Robert Horace Redfield, The generalized interval topology on distributive

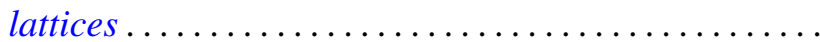

James Wilson Stepp, Algebraic maximal semilattices .... . .

Patrick Noble Stewart, A sheaf theoretic representation of rings with Boolean orthogonalities ........................

Ting-On To and Kai Wing Yip, A generalized Jensen's inequality......... 255

Arnold Lewis Villone, Second order differential operators with self-adjoint

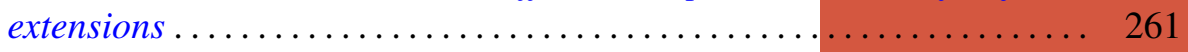

Martin E. Walter, On the structure of the Fourier-Stieltjes algebra ....... 267

John Wermer, Subharmonicity and hulls .................... 283

Edythe Parker Woodruff, A map of $E^{3}$ onto $E^{3}$ taking no disk onto a

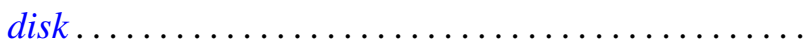

